\title{
Switching to criticality by synchronized input
} Anna Levina*1,2, Theo Geisel ${ }^{1,2}$ and J Michael Herrmann 1,2,3

Address: ${ }^{1}$ Bernstein Center for Computational Neuroscience, 37073 Göttingen, Germany, ${ }^{2}$ Max Planck Institute for Dynamics and SelfOrganization, 37073 Göttingen, Germany and ${ }^{3} \mathrm{IPAB}$, School of Informatics, University of Edinburgh, Edinburgh, EH8 9AB, UK

Email: Anna Levina* - anna.@nld.ds.mpg.de

* Corresponding author

from Eighteenth Annual Computational Neuroscience Meeting: CNS*2009

Berlin, Germany. 18-23 July 2009

Published: 13 July 2009

BMC Neuroscience 2009, I0(SuppI I):PI55 doi:10.1 I86/I47I-2202-I0-SI-PI55

This abstract is available from: http://www.biomedcentral.com/I47I-2202/I0/SI/PI55

(C) 2009 Levina et al; licensee BioMed Central Ltd.

The concept of self-organized criticality (SOC) describes a variety of phenomena ranging from plate tectonics, the dynamics of granular media and stick-slip motion to neural avalanches [1]. In all these cases the dynamics is mar-

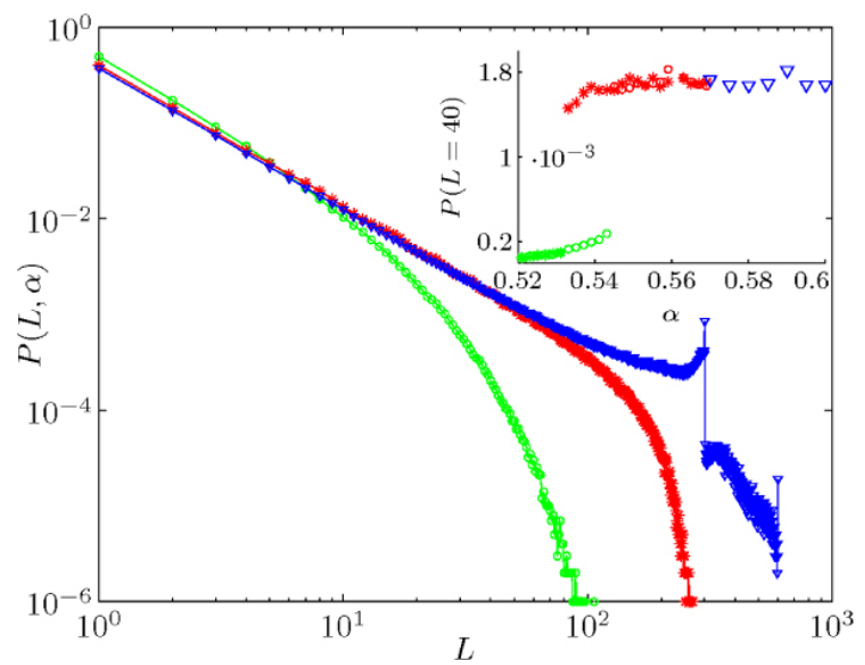

Figure I

The distribution of avalanche sizes changes in dependence on the interaction parameter $\alpha$ from subcritical (circles, $\alpha=0.52$ ) via critical (stars, $\alpha=$ 0.56 ) to supercritical (triangles, $\alpha=\mathbf{0 . 5 9}$ ). The inset shows the hysteresis of the distribution at an exemplary avalanche size $(L=40)$. The circles result at increasing $\alpha$, stars at decreasing $\alpha$. ginally stable and event sizes obey a characteristic powerlaw distribution.

It was previously shown that an extended critical interval can be obtained in a neural network by incorporation of depressive synapses [2]. In the present study we scrutinize a more realistic dynamics for the synaptic interactions that can be considered as the state-of-the-art in computational modeling of synaptic interaction (Figure 1) [2]. Interestingly, the more complex model does not exclude an analytical treatment and it shows a type of stationary state consisting of self-organized critical phase and a subcritical

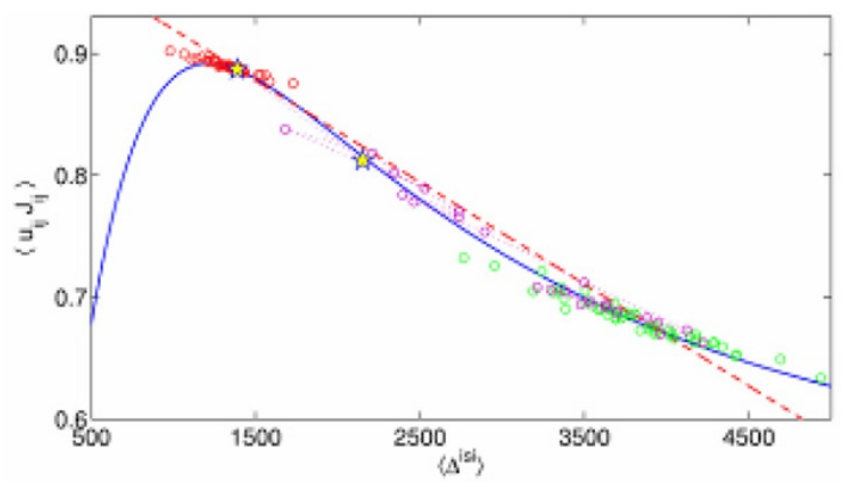

Figure 2

Dynamic transition from a critical (red circles) to a subcritical state (green circles) by short-term activity deprivation. The two stars represent the immediate effect of the deprivation in a particular trial. 
phase that has not been previously described. The phases are connected by first- or second-order phase transitions in a cusp bifurcation. Switching between phases can be induced by synchronized activity or by activity deprivation (Figure 2). We present exact analytical results supported by extensive numerical simulations.

By elucidating the relation between the elementary synaptic processes and the network dynamics, our mean-field approach revealed a macroscopic bifurcation pattern, which can be verified experimentally through predicted hysteresis. Furthermore it may be able to explain observations of up and down states in the prefrontal cortex [4] as well as the discrete changes in synaptic potentiation and depression [5] as network effects.

\section{References}

I. Beggs J, Plenz D: Neuronal avalanches are diverse and precise activity patterns that are stable for many hours in cortical slice cultures. J Neurosci 2004, 24:5216-5229.

2. Levina A, Herrmann JM, Geisel T: Dynamical synapses causing self-organized criticality in neural networks. Nature Physics 2007, 3:857-860.

3. Tsodyks MV, Markram H: The neural code between neocortical pyramidal neurons depends on neurotransmitter release probability. PNAS 1997, 94:7| 9-723.

4. Wilson CJ: The generation of natural firing patterns in neostriatal neurons. Prog Brain Res 1993, 99:277-297.

5. Petersen $\mathrm{CCH}$, Malenka RC, Nicoll RA, Hopfield J]: All-or-none potentiation at CA3-CAI synapses. PNAS Neurobiology 1998, 95:4732-4737.
Publish with Bio Med Central and every scientist can read your work free of charge

"BioMed Central will be the most significant development for disseminating the results of biomedical research in our lifetime. "

Sir Paul Nurse, Cancer Research UK

Your research papers will be:

- available free of charge to the entire biomedical community

- peer reviewed and published immediately upon acceptance

- cited in PubMed and archived on PubMed Central

- yours - you keep the copyright

Submit your manuscript here:

http://www.biomedcentral.com/info/publishing_adv.asp 